\title{
Ultrasonography for the Prediction of High-Volume Lymph Node Metastases in Papillary Thyroid Carcinoma: Should Surgeons Believe Ultrasound Results?
}

\author{
Chunhao Liu ${ }^{1}$ Lei Zhang ${ }^{2} \cdot$ Yuewu Liu ${ }^{1} \cdot$ Yu Xia $^{3} \cdot$ Yue Cao $^{1} \cdot$ Ziwen Liu $^{1} \cdot$ Ge Chen $^{1} \cdot$

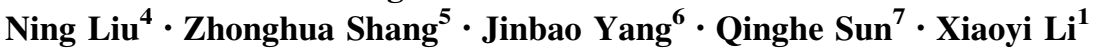

Accepted: 9 August 2020/Published online: 11 September 2020

(C) The Author(s) 2020

\begin{abstract}
Background Lymph node metastasis (LNM) often occurs in papillary thyroid carcinoma (PTC); the efficacy of ultrasound for predicting high-volume lymph node metastases (LNMs) in patients with PTC remains unexplored. Methods The medical records of 2073 consecutive PTC patients were reviewed. Sensitivity, specificity, positive predictive value (PPV) and negative predictive value (NPV) were calculated to evaluate the efficacy of ultrasound. Risk factors for LNM/high-volume LNMs and lymph node involvement on ultrasound (usLNM) were identified by univariate and multivariate analyses.

Results Of all the patients, 936 (45.2\%) patients had LNMs, and 254 (12.3\%) patients had high-volume LNMs. The sensitivity of ultrasound for detecting LNM/high-volume LNMs was $27.9 \%$ and $63.8 \%$, respectively; the specificity was $93.1 \%$ and $90.3 \%$, respectively. The NPV for ultrasound in detecting high-volume LNMs was $94.7 \%$. In multivariate analysis, male sex $(\mathrm{OR}=2.108, p<0.001)$, tumor diameter $>1.0 \mathrm{~cm}(\mathrm{OR}=2.304, p<0.001)$ and usLNM $(+)(\mathrm{OR}=12.553, p<0.001)$ were independent clinical risk factors for high-volume LNMs. Tumor diameter $>1 \mathrm{~cm}(\mathrm{OR}=3.036, p<0.001)$ and male sex $(\mathrm{OR}=1.642, p<0.001)$ were independent clinical risk factors for usLNM; a skilled sonographer (OR $=1.121, p=0.358)$ was not significantly associated with usLNM. Conclusions Lymph node involvement found by ultrasound has great predictive value for high-volume LNMs; the NPV is very high for patients without lymph node involvement on ultrasound. The ultrasound results do not appear to be influenced by the experience of the sonographer.
\end{abstract}

Chunhao Liu and Lei Zhang have contributed equally.

Electronic supplementary material The online version of this article (https://doi.org/10.1007/s00268-020-05755-0) contains supplementary material, which is available to authorized users.

Xiaoyi Li

li.xiaoyi@263.net

1 Department of General Surgery, Peking Union Medical College Hospital, Chinese Academy of Medical Sciences and Peking Union Medical College, No. 1 Shuai Fu Yuan, Dongcheng District, Beijing 100730, China

2 Department of Liver Surgery, Peking Union Medical College Hospital, Chinese Academy of Medical Sciences and Peking Union Medical College, Beijing 100730, China
3 Department of Ultrasound, Peking Union Medical College Hospital, Chinese Academy of Medical Sciences and Peking Union Medical College, Beijing 100730, China

4 McKusick-Zhang Center for Genetic Medicine, State Key Laboratory of Medical Molecular Biology, Institute of Basic Medical Sciences, Chinese Academy of Medical Sciences and Peking Union Medical College, Beijing 100730, China

5 Department of General Surgery, Second Hospital of Shanxi Medical University, Taiyuan 030001, Shanxi Province, China

6 Department of General Surgery, People's Liberation Army Bethune International Peace Hospital, Shijiazhuang 050082, Hebei Province, China

7 Department of General Surgery, Cangzhou People's Hospital, Cangzhou 061000, Hebei Province, China 


$\begin{array}{ll}\text { Abbreviations } \\ \text { LNM } & \text { Lymph node metastasis } \\ \text { LNMs } & \text { Lymph node metastases } \\ \text { PTC } & \text { Papillary thyroid carcinoma } \\ \text { PTMC } & \text { Papillary thyroid microcarcinoma } \\ \text { LND } & \text { Lymph node dissection } \\ \text { PPV } & \text { Positive predictive value } \\ \text { NPV } & \text { Negative predictive value } \\ \text { usLNM } & \text { Lymph node involvement on ultrasound }\end{array}$

\section{Introduction}

Papillary thyroid carcinoma (PTC) accounts for approximately $85 \%$ of thyroid malignancies. The incidence has been increasing rapidly in recent years, and it has even become the highest of all malignancies in Korea [1-3]. With appropriate treatment, the prognosis of PTC is satisfactory, and the 10-year overall survival rate is over $90 \%$ [4]. Throughout the lifespan, the main problems confronted by most patients are recurrence and reoperation.

Cervical lymph node metastases (LNMs) are very common, especially in the central compartment, and the metastatic rate is 20-90\% [5]. The prognostic significance and surgical management of LNM remain controversial. However, several studies have shown that LNM is the second independent risk factor for PTC patients following distant metastasis. Moreover, for patients with high-volume LNMs ( $>5$ pathological lymph node metastases), the risk of recurrence increases significantly [6,7]. Based on the 2015 ATA risk stratification system, patients with highvolume LNMs have an intermediate risk of recurrence, and treatment for these patients should thus be highly proactive [8]. Therefore, identifying patients with high-volume LNMs before surgery is of great significance for treatment decision-making.

Ultrasound is the most convenient and effective method for screening thyroid disease. However, ultrasound is operator dependent and cannot image deep anatomic structures that are adequately shadowed by bone or air. Although studies have noted that the efficacy of lymph node metastasis detection by ultrasound is not ideal $[9,10]$, the preoperative detection of high-volume LNMs by ultrasound remains unexplored. This study was designed to evaluate the value of preoperative ultrasound for predicting high-volume LNMs in patients with papillary thyroid carcinoma.

\section{Patients and methods}

This retrospective study included a total of 2073 consecutive PTC patients (male: 490, female: 1583) who underwent primary surgery in our institution for 1 year. This study was approved by the Ethics Committees of Peking Union Medical College Hospital, and all the patients had signed the informed consent.

The inclusion criteria were as follows: (1) newly diagnosed PTC and (2) underwent lobectomy or near-total/total thyroidectomy with cervical lymph node dissection (LND) according to the Chinese guidelines (All PTC patients should undergo at least ipsilateral central neck dissection and lateral neck dissection when $\mathrm{cN} 1 \mathrm{~b}$ was indicated. The recommendation for primary thyroid surgery is similar to ATA guidelines) [11]. The exclusion criteria were as follows: (1) underwent revision surgery; (2) not underwent cervical LND; and (3) non-PTC on pathological examination. In multifocal cases, the largest diameter of tumors was included for analysis. The number of metastatic lymph nodes was calculated by pathological examination. More than 5 metastatic lymph nodes were defined as high-volume LNMs.

Preoperative ultrasound, which was performed in all patients, provided data on the extent of thyroid disease and suspicious cervical central and lateral lymphadenopathy. There are six features which were used for judging clinically involved nodes (cN1) in our institution. They are enlargement and round shape, loss of the fatty hilum, peripheral vascularity, hyperechogenicity, cystic aspect and microcalcifications. The criteria for lymph nodes involvement are any two or more of the first three features, or any one or more of the last three features, when chronic lymphocytic thyroiditis was considered on ultrasound examination; the criteria for lymph nodes involvement are any one or more of the six features when chronic lymphocytic thyroiditis wasn't considered. In terms of analyzing the influence of the sonographer on the examination results, sonographers were divided into two groups based on their experience: group A, general sonographer who had less than 5 years experience in thyroid ultrasound with approximately 600-700 cases per year, and group B, skilled thyroid sonographer who had performed over 1000 thyroid screening cases per year over the past 5 years.

In this study, we compared the preoperative ultrasound results and postoperative pathological LNM to determine the accuracy of the ultrasound diagnosis of lymph node metastasis. The ultrasound findings of lymph node involvement as a criterion for suggesting high-volume LNMs were used to evaluate the efficacy of ultrasound diagnosis for high-volume lymph node metastases. We also analyzed the clinical factors associated with pathological 
lymph node metastasis, high-volume lymph node metastases and lymph node involvement on ultrasound.

Statistical analysis was performed using SPSS software (version 25.0). For sensitivity and specificity evaluations, the following formulas were applied using the histopathological report as the gold standard: sensitivity = true positives/(true positives + false negatives) and specificity $=$ true negatives/(true negatives + false positives). Positive predictive value $=$ true positives $/$ (true positives + false positives) and negative predictive value $=$ true negatives/(true negatives + false negatives) . Fisher's exact test or the $\chi^{2}$ test was used to examine the differences between patients with and without LNM/highvolume LNM. Multivariate models were used to determine the risk factors for LNM/high-volume LNMs; $p$ value $<0.05$ was considered to indicate statistical significance.

\section{Results}

\section{Clinicopathological backgrounds}

A total of 2073 PTC patients were included in the study for analysis (male: 490, female: 1583). Of all the patients, 936 (45.2\%) patients had LNM, and 254 (12.3\%) patients had high-volume LNMs. A total of 1414 cases were papillary thyroid microcarcinoma (PTMC), $513(36.3 \%)$ patients had LNM, and 96 (6.8\%) patients had high-volume LNMs (Table 1).

\section{Efficacy of preoperative ultrasound for LNM and high-volume LNMs}

A total of $339(16.4 \%)$ patients showed lymph node involvement on preoperative ultrasound, of whom 261 (77.0\%) had LNM confirmed by postoperative pathology; a total of 1734 (83.6\%) patients showed no lymph node involvement on preoperative ultrasound, of whom 1137 (65.6\%) had no LNM confirmed by postoperative pathology. Among the PTMC patients, a total of 157 (11.1\%) showed lymph node involvement on preoperative ultrasound, of whom 101 (64.3\%) had LNM confirmed by postoperative pathology; a total of 1257 (88.9\%) patients showed no lymph node involvement on preoperative ultrasound, of whom 845 (67.2\%) had no LNM confirmed by postoperative pathology. The sensitivity of preoperative ultrasound for detecting LNM was $27.9 \%$ (261/936), the specificity was $93.1 \%(1059 / 1137)$, the negative predictive value (NPV) was $61.1 \%(1059 / 1734)$, and the accuracy rate was $63.7 \%(1320 / 2073)$ (Table 2).

Among 339 (16.4\%) PTC patients with lymph node involvement on preoperative ultrasound, 162 (47.8\%) patients had confirmed high-volume LNMs by postoperative pathology; among $1734(83.6 \%)$ patients with no lymph node involvement on preoperative ultrasound, 1642 (94.7\%) patients had confirmed absence of high-volume LNMs by postoperative pathology. Among 157 (11.1\%) PTMC patients with lymph node involvement on preoperative ultrasound, 55 (35.0\%) patients had confirmed highvolume LNMs by postoperative pathology; among 1257 $(88.9 \%)$ patients with no lymph node involvement on preoperative ultrasound, 1216 (96.7\%) patients had confirmed absence of high-volume LNMs by postoperative pathology. The sensitivity of preoperative ultrasound for predicting high-volume LNMs was $63.8 \%$ (162/254), the specificity was $90.3 \%(1642 / 1819)$, the negative predictive value (NPV) was $94.7 \%$ (1642/1734), and the accuracy rate was $87.0 \%(1804 / 2073)$ (Table 2).

\section{Univariate and multivariate analyses for LNM}

Univariate analysis showed that compared with control group (patients without LNM), LNM were more common in male sex $(58.4 \%$ vs. $41.1 \%, p<0.001)$, age $<55$ years old $(47.0 \%$ vs. $34.9 \%, p<0.001)$, tumor diameter $>1 \mathrm{~cm}$ $(64.2 \%$ vs. $36.3 \%, p<0.001)$ and usLNM (+) patients (77.0\% vs. $38.9 \%, p<0.001$ ) (Table 3). Multivariate analysis showed that male sex $(\mathrm{OR}=1.869,95 \% \mathrm{CI}$ 1.499-2.331, $\quad p<0.001)$, tumor diameter $>1.0 \mathrm{~cm}$ $(\mathrm{OR}=2.203, \quad 95 \%$ CI 1.892-2.565, $p<0.001)$ and usLNM $\quad(+) \quad(\mathrm{OR}=3.978, \quad 95 \% \quad$ CI $2.999-5.275$, $p<0.001)$ were independent risk factors for LNM. In contrast, age $>55$ years old $(\mathrm{OR}=0.605,95 \% \quad \mathrm{CI}$ 0.462-0.791, $p<0.001)$ was an independent protective factor for LNM (Table 4).

Univariate analysis was used to evaluate the differences between patients with (254 cases) and without high-volume LNMs (1819 cases). Results showed that compared with control group (patients without high-volume LNMs), highvolume LNMs were more common in male sex $(20.2 \%$ vs. $9.8 \%, p<0.001)$, age $\leq 55$ years old $(12.9 \%$ vs. $8.8 \%$, $p<0.001)$, tumor diameter $>1 \mathrm{~cm}(24.0 \%$ vs. $6.8 \%$, $p<0.001)$ and usLNM (+) patients $(47.8 \%$ vs. $5.3 \%$, $p<0.001$ ) (Table 3). Multivariate analysis showed that male sex $(\mathrm{OR}=2.108,95 \%$ CI 1.517-2.929, $p<0.001)$, tumor diameter $>1.0 \mathrm{~cm} \quad(\mathrm{OR}=2.304, \quad 95 \% \quad \mathrm{CI}$ 1.889-2.811, $p<0.001)$ and usLNM $(+)(\mathrm{OR}=12.553$, 95\% CI 9.181-17.165, $p<0.001)$ were independent risk factors for high-volume LNMs (Table 4).

\section{Univariate and multivariate analyses for usLNM}

The number of patients in group A and B was 1251 and 822 , respectively. There were no significant differences in clinicopathologic features between the two groups 
Table 1 Patient demographics

\begin{tabular}{|c|c|c|}
\hline Item & PTC & PTMC \\
\hline \multicolumn{3}{|l|}{ Sex } \\
\hline Female & $1583(76.4)$ & $1098(77.7)$ \\
\hline Male & $490(23.6)$ & $316(22.3)$ \\
\hline \multicolumn{3}{|l|}{ Age } \\
\hline$\leq 55$ & $1755(84.7)$ & $1200(84.9)$ \\
\hline$>55$ & $318(15.3)$ & $214(15.1)$ \\
\hline \multicolumn{3}{|l|}{ BMI } \\
\hline 18.5 & $55(2.7)$ & $37(2.6)$ \\
\hline$<18.5-24$ & $970(46.8)$ & $664(47.0)$ \\
\hline$<24-28$ & $755(36.4)$ & $511(36.1)$ \\
\hline $28 \sim$ & $293(14.1)$ & $202(14.3)$ \\
\hline \multicolumn{3}{|l|}{ Tumor diameter ${ }^{\mathrm{a}}$} \\
\hline$\leq 1 \mathrm{~cm}$ & $1414(68.2)$ & - \\
\hline$\leq 0.5$ & - & $315(22.3)$ \\
\hline$>0.5-1.0$ & - & $1099(77.7)$ \\
\hline$>1.0-2.0$ & $499(24.1)$ & - \\
\hline$>2.0$ & $160(7.7)$ & - \\
\hline \multicolumn{3}{|l|}{ usLNM } \\
\hline Yes & $339(16.4)$ & $157(11.1)$ \\
\hline No & $1734(83.6)$ & $1257(88.9)$ \\
\hline \multicolumn{3}{|l|}{ Operation } \\
\hline Hemithyroidectomy + LND & $410(19.8)$ & $342(24.1)$ \\
\hline Total thyroidectomy + LND & $1663(80.2)$ & $1072(75.9)$ \\
\hline \multicolumn{3}{|l|}{ Multilfocality } \\
\hline Yes & $731(35.3)$ & $457(32.3)$ \\
\hline No & $1342(64.7)$ & $957(67.7)$ \\
\hline \multicolumn{3}{|l|}{ Capsule invasion } \\
\hline Yes & $643(31.0)$ & $320(22.6)$ \\
\hline No & $1430(69.0)$ & $1094(77.4)$ \\
\hline \multicolumn{3}{|l|}{ Chronic lymphocytic thyroiditis } \\
\hline Yes & $509(24.6)$ & $337(23.8)$ \\
\hline No & $1564(75.4)$ & $1077(76.2)$ \\
\hline \multicolumn{3}{|l|}{ LNM } \\
\hline Yes & $936(45.2)$ & $513(36.3)$ \\
\hline No & $1137(54.8)$ & $901(63.7)$ \\
\hline \multicolumn{3}{|l|}{ hvLNMs } \\
\hline Yes & $254(12.3)$ & $96(6.8)$ \\
\hline No & 1819 (87.7) & $1318(93.2)$ \\
\hline
\end{tabular}

$B M I$ body mass index, usLNM lymph node involvement on ultrasound, $L N D$ lymph node dissection, $L N M$ lymph node metastasis, $h v L N M s$ high-volume lymph node metastases

${ }^{\mathrm{a}}$ Diameter of the largest lesion in multifocal tumors

(Supplementary Table). Univariate analysis showed that usLNM was more common in male sex $(22.4 \%$ vs. $14.5 \%$, $p<0.001)$, age $\leq 55$ years old $(17.2 \%$ vs. $11.9 \%$, $p=0.021)$ and tumor diameter $>1 \mathrm{~cm}$ patients $(27.6 \%$ vs. $11.1 \%, p<0.001)$ (Table 5). Multivariate analysis showed that male sex $(\mathrm{OR}=1.642,95 \%$ CI $1.264-2.133$, $p<0.001)$ and tumor diameter $>1 \mathrm{~cm}(\mathrm{OR}=3.036,95 \%$ CI 2.389-3.859, $p<0.001)$ were independent risk factors for usLNM. In contrast, age $>55$ years old $(\mathrm{OR}=0.646$, 95\% CI $0.446-0.935, p=0.02$ ) was an independent protective factor for usLNM, and a skilled sonographer $(\mathrm{OR}=1.121,95 \%$ CI $0.879-1.429, p=0.358)$ was not significantly associated with usLNM (Table 6).

\section{Discussion}

Our study showed that the sensitivity of LNM detection for preoperative ultrasound was relatively low: $27.9 \%$ for PTC and $19.7 \%$ for PTMC. However, the sensitivity of highvolume LNMs detection was significantly increased (63.8\%); furthermore, the NPV could reach $94.7 \%$ for PTC and $96.7 \%$ for PTMC. Obviously, the impact of the diagnostic efficacy of preoperative ultrasound on the clinical diagnosis and treatment of PTC is worthy of deep discussion.

An increase in the incidence of thyroid cancer, especially PTC, has been reported in several countries [12, 13], including China [14], over the past several decades. Although the overall survival of PTC patients is better than that of patients with other cancers, regional LNMs are frequently observed in PTC [15]. The lymph node status affects decision-making in terms of treatment and prognosis in patients with PTC [16]. Ultrasound has been the first choice of imaging modality for the preoperative assessment of LNM. However, the sensitivity of ultrasound for detecting LNM of the central neck was only 33\%, and the specificity was $93 \%$; for the lateral compartment, the sensitivity and specificity were $70 \%$ and $84 \%$, respectively [17]. Preoperative ultrasound only detects half of the lymph nodes found during surgery [18]. Our data also showed that preoperative ultrasound had poor diagnostic accuracy for assessing cervical LNM in PTC, and the sensitivity, specificity and accuracy of preoperative ultrasound for cervical LNM were $27.9 \%, 93.1 \%$ and $63.7 \%$, respectively. In the face of this diagnostic efficacy for LNM, are the results of ultrasound still worth trusting?

The failure of preoperative ultrasound to detect metastatic lesions may be common. However, these lymph node involvements that cannot be showed on ultrasound may be representative of fewer and smaller lesions and may not have a significant influence on treatment outcomes. In regions routinely performing prophylactic LND, the LNM rate of PTMC can reach 30-40\% [19, 20], but it has been reported to be only $3.2 \%$ in regions without routine prophylactic LND. However, the postoperative recurrence and reoperation rate of the two groups did not seem to have an obvious difference $[20,21]$. The 2015 ATA guidelines 
Table 2 Accuracy evaluation of ultrasound prediction of lymph node metastases

\begin{tabular}{|c|c|c|c|c|c|c|}
\hline \multirow[t]{2}{*}{ Item } & \multicolumn{2}{|l|}{ PTMC $n=1414$} & \multicolumn{2}{|c|}{ Non-PTMC $n=659$} & \multicolumn{2}{|l|}{ PTC $n=2073$} \\
\hline & LNM & hvLNMs & LNM & hvLNMs & LNM & hvLNMs \\
\hline Sensitivity & $19.7 \%(101 / 513)$ & $57.3 \%(55 / 96)$ & $37.8 \%(160 / 423)$ & $67.7 \%(107 / 158)$ & $27.9 \%(261 / 936)$ & $63.8 \%(162 / 254)$ \\
\hline Specificity & $93.8 \%(845 / 901)$ & $92.3 \%(1216 / 1318)$ & $90.7 \%(214 / 236)$ & $85.0 \%(426 / 501)$ & $93.1 \%(1059 / 1137)$ & $90.3 \%(1642 / 1819)$ \\
\hline PPV & $64.3 \%(101 / 157)$ & $35.0 \%(55 / 157)$ & $87.9 \%(160 / 182)$ & $58.8 \%(107 / 182)$ & $77.0 \%(261 / 339)$ & $47.8 \%(162 / 339)$ \\
\hline NPV & $67.2 \%(845 / 1257)$ & $96.7 \%(1216 / 1257)$ & $44.9 \%(214 / 477)$ & $89.3 \%(426 / 477)$ & $61.1 \%(1059 / 1734)$ & $94.7 \%(1642 / 1734)$ \\
\hline Accuracy & $66.9 \%(946 / 1414)$ & $89.9 \%(1271 / 1414)$ & $56.8 \%(374 / 659)$ & $80.9 \%(533 / 659)$ & $63.7 \%(1320 / 2073)$ & $87.0 \%(1804 / 2073)$ \\
\hline
\end{tabular}

$L N M$ lymph node metastasis, $h v L N M s$ high-volume lymph node metastases, $P P V$ positive predictive value, $N P V$ negative predictive value

Table 3 Univariate analysis of risk factors for pathological LNM and hvLNMs

\begin{tabular}{|c|c|c|c|c|c|c|}
\hline Item & $\begin{array}{l}\operatorname{LNM}(-) \\
n=1137(\%)\end{array}$ & $\begin{array}{l}\operatorname{LNM}(+) \\
n=936(\%)\end{array}$ & $P$ value & $\begin{array}{l}\text { hvLNMs (-) } \\
n=1819(\%)\end{array}$ & $\begin{array}{l}\text { hvLNMs (+) } \\
n=254(\%)\end{array}$ & $P$ value \\
\hline Sex & & & $<0.001$ & & & $<0.001$ \\
\hline Female & 933 (58.9) & $650(41.1)$ & & $1428(90.2)$ & $155(9.8)$ & \\
\hline Male & 204 (41.6) & $286(58.4)$ & & 391 (79.8) & 99 (20.2) & \\
\hline Age & & & $<0.001$ & & & 0.04 \\
\hline$\leq 55$ & $930(53.0)$ & $825(47.0)$ & & $1529(87.1)$ & 226 (12.9) & \\
\hline$>55$ & $207(65.1)$ & $111(34.9)$ & & $290(91.2)$ & $28(8.8)$ & \\
\hline BMI & & & 0.950 & & & 0.685 \\
\hline$\sim 18.5$ & $32(58.2)$ & $23(41.8)$ & & $50(90.9)$ & $5(9.1)$ & \\
\hline$<18.5-24$ & $534(55.1)$ & $436(44.9)$ & & 851 (87.7) & $119(12.3)$ & \\
\hline$<24-28$ & $410(54.3)$ & 345 (45.7) & & $666(88.2)$ & 89 (11.8) & \\
\hline $28 \sim$ & $161(54.9)$ & $132(45.2)$ & & $252(86.0)$ & $41(14.0)$ & \\
\hline Tumor diameter ${ }^{\mathrm{a}}$ & & & $<0.001$ & & & $<0.001$ \\
\hline$\leq 1 \mathrm{~cm}$ & 901 (63.7) & $513(36.3)$ & & 1318 (93.2) & $96(6.8)$ & \\
\hline$>1 \mathrm{~cm}$ & $236(35.8)$ & $423(64.2)$ & & $501(76.0)$ & $158(24.0)$ & \\
\hline usLNM & & & $<0.001$ & & & $<0.001$ \\
\hline Yes & $78(23.0)$ & $261(77.0)$ & & $177(52.2)$ & $162(47.8)$ & \\
\hline No & 1059 (61.1) & 675 (38.9) & & $1642(94.7)$ & $92(5.3)$ & \\
\hline
\end{tabular}

$B M I$ body mass index, usLNM lymph node involvement on ultrasound, LNM lymph node metastasis, hvLNMs high-volume lymph node metastases

${ }^{\mathrm{a}}$ Diameter of the largest lesion in multifocal tumors

emphasized the impact of the size and number of metastatic lesions on prognosis. The recurrence rate of patients with high-volume LNMs ( $>5$ metastatic lymph nodes) was $19 \%$, which was significantly higher than the recurrence rate of $2-4 \%$ in $\mathrm{cN} 0 \mathrm{pN} 1$ patients $(<5$ metastatic lymph nodes) [8]. These patients with high-volume LNMs should be identified and provided treatment cautiously, as their treatment outcomes have a great impact on the prognosis of disease. Based on our results, when lymph node involvement was detected by ultrasound, the rate of postoperative high-volume LNMs was 47.8\%; when lymph node involvement was not detected, the rate of postoperative high-volume LNMs was 5.3\%. The sensitivity, specificity and accuracy of the diagnosis were $63.8 \%, 90.3 \%$ and $87.0 \%$, respectively. These findings indicate that lymph node involvement found by ultrasound has great value for predicting postoperative high-volume LNMs. Furthermore, when no lymph node involvement was detected by preoperative ultrasound, the negative predictive value for high-volume LNMs was $94.7 \%$, which was much higher for PTMC (96.7\%). The results suggest that the possibility of high-volume LNMs is very low for patients without lymph node involvement on preoperative ultrasound, and less aggressive treatment (active surveillance or treatment without prophylactic LND) may not bring poor outcomes for these patients. 
Table 4 Multivariate analysis of risk factors for pathological LNM and hvLNMs

\begin{tabular}{lrlr}
\hline Item & OR & $95 \%$ CI & $P$ value \\
\hline LNM & & & \\
usLNM (+) & 3.978 & $2.999-5.275$ & $<0.001$ \\
Tumor diameter ${ }^{\mathrm{a}}>1 \mathrm{~cm}$ & 2.203 & $1.892-2.565$ & $<0.001$ \\
Male & 1.869 & $1.499-2.331$ & $<0.001$ \\
BMI $\geq 24$ & 1.015 & $0.842-1.225$ & 0.873 \\
Age $>55$ & 0.605 & $0.462-0.791$ & $<0.001$ \\
huLNMs & & & \\
usLNM (+) & 12.553 & $9.181-17.165$ & $<0.001$ \\
Tumor diameter ${ }^{\mathrm{a}}>1 \mathrm{~cm}$ & 2.304 & $1.889-2.811$ & $<0.001$ \\
Male & 2.108 & $1.517-2.929$ & $<0.001$ \\
BMI $\geq 24$ & 1.034 & $0.757-1.414$ & 0.832 \\
Age $>55$ & 0.728 & $0.449-1.181$ & 0.119 \\
\hline
\end{tabular}

$O R$ odds ratio, $C I$ confidence interval, $B M I$ body mass index, usLNM lymph node involvement on ultrasound, $L N M$ lymph node metastasis, hvLNMs high-volume lymph node metastases

${ }^{\mathrm{a}}$ Diameter of the largest lesion in multifocal tumors

Table 5 Univariate analysis of risk factors for usLNM

\begin{tabular}{lccc}
\hline Item & $\begin{array}{c}\text { usLNM (-) } \\
n=1734(\%)\end{array}$ & $\begin{array}{r}\text { usLNM }(+) \\
n=339(\%)\end{array}$ & $P$ value \\
\hline Sex & & & $<0.001$ \\
$\quad$ Female & $1354(85.5)$ & $155(14.5)$ & \\
$\quad$ Male & $391(77.6)$ & $99(22.4)$ & \\
Age & & & 0.021 \\
$\quad \leq 55$ & $1454(82.8)$ & $301(17.2)$ & \\
$>55$ & $280(88.1)$ & $38(11.9)$ & \\
BMI & & & \\
$\sim 18.5$ & $48(87.3)$ & $7(12.7)$ & \\
$<18.5-24$ & $806(83.1)$ & $164(16.9)$ & \\
$<24-28$ & $634(84.0)$ & $121(16.0)$ & \\
$28-$ & $246(84.0)$ & $47(16.0)$ & \\
Tumor diameter ${ }^{\mathrm{a}}$ & & & \\
$\quad$ & & & \\
$>1$ cm & $1257(88.9)$ & $157(11.1)$ & \\
$>1$ cm & $477(72.4)$ & $182(27.6)$ & \\
Sonographer & & & \\
$\quad$ Group A & $1054(84.3)$ & $197(15.7)$ & \\
Group B & $680(82.7)$ & $142(17.3)$ & \\
\hline
\end{tabular}

$B M I$ body mass index, $u s L N M$ lymph node involvement on ultrasound ${ }^{a}$ Diameter of the largest lesion in multifocal tumors; A general sonographer; B skilled sonographer

Our results showed that among the clinical factors that may affect the prediction of high-volume LNMs, male sex
(20.2\% vs. $9.8 \%, p<0.001)$, age $\leq 55$ years old $(12.9 \%$ vs. $8.8 \%, p<0.001)$ and tumor diameter $>1 \mathrm{~cm}(24.0 \%$ vs. $6.8 \%, p<0.001)$ were risk factors for high-volume LNMs, results that were similar to those of previous studies $[22,23]$. Nevertheless, the risk factor for high-volume LNMs, which was more important than these known clinical risk factors, was lymph node involvement detected by preoperative ultrasound with an OR of 12.553. Ultrasound evaluation is an operator-dependent method [24]. Surgeons should be particularly vigilant regarding whether the experience of the sonographer will affect the ultrasound results, especially in the evaluation of cervical lymph node involvement, which has good predictive value for highvolume LNMs. Our results showed that the proportion of LNM detected by skilled and general sonographers was $17.3 \%$ and $15.7 \%(p=0.363)$ in the two groups with similar conditions, respectively (Supplementary Table). In the multivariate analysis, a skilled sonographer was not an independent risk factor for detecting LNM by ultrasound $(\mathrm{OR}=1.121,95 \%$ CI $0.879-1.429, p=0.358)$. However, this study was not a standard diagnostic test; therefore, a standard diagnostic test should be conducted to clarify the impact of sonographer experience on ultrasound results. Moreover, there are needs to expand the population and conduct a multi-center prospective study to evaluate the value of ultrasound in predicting high-volume lymph node metastases for the results just from a retrospective study in one center.

\section{Conclusion}

Although preoperative ultrasound has poor sensitivity for detecting LNM, lymph node involvement found by ultrasound has great predictive value for high-volume LNMs; in particular, the NPV is very high for patients without lymph node involvement on preoperative ultrasound. Therefore, it is acceptable to adopt less aggressive treatment strategies for these patients. Moreover, the use of preoperative ultrasound for predicting high-volume lymph node metastases does not appear to be influenced by the experience of the sonographer.

Acknowledgements The authors thank Mr. Yanlong Li for statistical guidance.

Funding This study was supported by the Chinese Academy of Medical Sciences (CAMS) Innovation Fund for Medical Sciences (CIFMS) (Grant No. 2016-I2M-1-002).

\section{Compliance with ethical standards}

Conflict of interest All authors declare that they have no conflict of interest. 
Table 6 Multivariate analysis of risk factors for usLNM

\begin{tabular}{lllc}
\hline Item & OR & $95 \%$ CI & $P$ value \\
\hline Male & 1.642 & $1.264-2.133$ & $<0.001$ \\
Age $>55$ & 0.646 & $0.446-0.935$ & 0.02 \\
Tumor diameter $^{\mathrm{a}}>1 \mathrm{~cm}$ & 3.036 & $2.389-3.859$ & $<0.001$ \\
BMI $\geq 24$ & 0.929 & $0.715-1.208$ & 0.583 \\
Sonographer B & 1.121 & $0.879-1.429$ & 0.358
\end{tabular}

OR odds ratio, $C I$ confidence interval, $B M I$ body mass index, $u s L N M$ lymph node involvement on ultrasound

${ }^{a}$ Diameter of the largest lesion in multifocal tumors; B skilled sonographer

Human and/or animal rights The study was approved by the Ethics Committees of Peking Union Medical College Hospital.

Informed consent Informed consent was obtained from all individual participants included in the study.

Open Access This article is licensed under a Creative Commons Attribution 4.0 International License, which permits use, sharing, adaptation, distribution and reproduction in any medium or format, as long as you give appropriate credit to the original author(s) and the source, provide a link to the Creative Commons licence, and indicate if changes were made. The images or other third party material in this article are included in the article's Creative Commons licence, unless indicated otherwise in a credit line to the material. If material is not included in the article's Creative Commons licence and your intended use is not permitted by statutory regulation or exceeds the permitted use, you will need to obtain permission directly from the copyright holder. To view a copy of this licence, visit http://creativecommons. org/licenses/by/4.0/.

\section{References}

1. Ahn HS, Kim HJ, Welch HG (2014) Korea's thyroid-cancer "epidemic"-screening and overdiagnosis. N Engl J Med 371:1765-1767

2. Davies L, Welch HG (2014) Current thyroid cancer trends in the United States. JAMA Otolaryngol Head Neck Surg 140:317-322

3. Fagin JA, Wells SA Jr (2016) Biologic and clinical perspectives on thyroid cancer. N Engl J Med 375:1054-1067

4. Sipos JA, Mazzaferri EL (2010) Thyroid cancer epidemiology and prognostic variables. Clin Oncol (R Coll Radiol) 22:395-404

5. Wang LY, Ganly I (2016) Nodal metastases in thyroid cancer: prognostic implications and management. Future Oncol 12:981-994

6. Randolph GW, Duh QY, Heller KS et al (2012) The prognostic significance of nodal metastases from papillary thyroid carcinoma can be stratified based on the size and number of metastatic lymph nodes, as well as the presence of extranodal extension. Thyroid 22:1144-1152

7. Lee J, Song Y, Soh EY (2014) Prognostic significance of the number of metastatic lymph nodes to stratify the risk of recurrence. World J Surg 38:858-862. https://doi.org/10.1007/ s00268-013-2345-6

8. Haugen BR, Alexander EK, Bible KC et al (2016) 2015 American Thyroid Association management guidelines for adult patients with thyroid nodules and differentiated thyroid cancer: the American Thyroid Association guidelines task force on thyroid nodules and differentiated thyroid cancer. Thyroid 26:1-133

9. Machado MR, Tavares MR, Buchpiguel CA et al (2017) Ultrasonographic evaluation of cervical lymph nodes in thyroid cancer. Otolaryngol Head Neck Surg 156:263-271

10. Ahn JE, Lee JH, Yi JS et al (2008) Diagnostic accuracy of CT and ultrasonography for evaluating metastatic cervical lymph nodes in patients with thyroid cancer. World J Surg 32:1552-1558. https://doi.org/10.1007/s00268-008-9588-7

11. Chinese Medical Association SOE et al (2012) Guidelines for the diagnosis and management of thyroid nodules and differentiated thyroid cancer. Chin J Endocrinol Metab 28(10):779-797

12. Morris LG, Tuttle RM, Davies L (2016) Changing trends in the incidence of thyroid cancer in the United $<$ States. JAMA Otolaryngol Head Neck Surg 142:709-711

13. Aschebrook-Kilfoy B, Kaplan EL, Chiu BC et al (2013) The acceleration in papillary thyroid cancer incidence rates is similar among racial and ethnic groups in the United States. Ann Surg Oncol 20:2746-2753

14. Zheng R, Zeng H, Zhang S et al (2017) Estimates of cancer incidence and mortality in China, 2013. Chin J Cancer 36:66

15. Liu W, Cheng R, Su Y et al (2017) Risk factors of central lymph node metastasis of papillary thyroid carcinoma: a single-center retrospective analysis of 3273 cases. Medicine (Baltimore) 96:e8365

16. Adam MA, Pura J, Goffredo P et al (2015) Presence and number of lymph node metastases are associated with compromised survival for patients younger than age 45 years with papillary thyroid cancer. J Clin Oncol 33:2370-2375

17. Zhao H, Li H (2019) Meta-analysis of ultrasound for cervical lymph nodes in papillary thyroid cancer: diagnosis of central and lateral compartment nodal metastases. Eur J Radiol 112:14-21

18. Leboulleux S, Girard E, Rose M et al (2007) Ultrasound criteria of malignancy for cervical lymph nodes in patients followed up for differentiated thyroid cancer. J Clin Endocrinol Metab 92:3590-3594

19. Zhang L, Liu H, Xie Y et al (2016) Risk factors and indication for dissection of right paraesophageal lymph node metastasis in papillary thyroid carcinoma. Eur J Surg Oncol 42:81-86

20. Lee J, Song Y, Soh EY (2014) Central lymph node metastasis is an important prognostic factor in patients with papillary thyroid microcarcinoma. J Korean Med Sci 29:48-52

21. Gschwandtner E, Klatte T, Swietek N et al (2016) Increase of papillary thyroid microcarcinoma and a plea for restrictive treatment: a retrospective study of 1391 prospective documented patients. Surgery 159:503-511

22. Wu X, Li B, Zheng C et al (2018) Risk factors for central lymph node metastases in patients with papillary thyroid microcarcinoma. Endocr Pract 24:1057-1062

23. Cheng F, Chen Y, Zhu L et al (2019) Risk factors for cervical lymph node metastasis of papillary thyroid microcarcinoma: a single-center retrospective study. Int J Endocrinol 2019:8579828

24. Lee HJ, Yoon DY, Seo YL et al (2018) Intraobserver and interobserver variability in ultrasound measurements of thyroid nodules. J Ultrasound Med 37:173-178

Publisher's Note Springer Nature remains neutral with regard to jurisdictional claims in published maps and institutional affiliations. 